\title{
The matrix remodelled
}

Biological tissues are held together by the extracellular matrix (ECM), a scaffold of fibre-like macromolecules that can be arranged in different ways, including curvy, isotropic and aligned networks (pictured). These arrangements help to support tissue function - for example, tendons have a highly aligned ECM that transmits tensile forces while avoiding injury but they can be altered by diseases such as cancer, which can transform isotropic ECM into anisotropic, aligned structures. How exactly the patterns are formed and remodelled has never been fully explained. Now, writing in a pair of papers in Nature
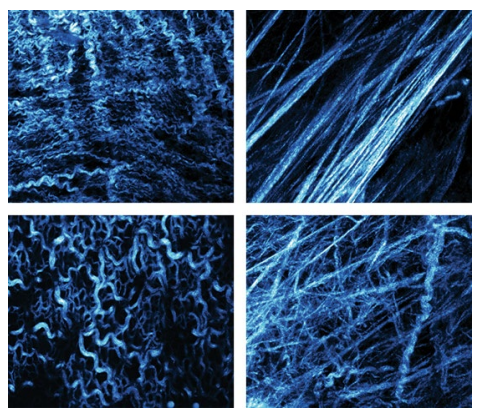

Credit: Adapted from Park, D. et al. Nat. Mater. 10.1038/s41563-019-0504-3 (2019), Springer Nature Limited
Materials and PLOS Computational Biology, Paul Bates, Erik Sahai and co-workers have demonstrated the role of feedback between the matrix and fibroblasts - the cells that deposit and maintain matrix fibres - in generating diverse ECM patterns.

Fibroblasts prefer to align along matrix fibres. Furthermore, when fibroblasts are in the presence of the matrix, they can form long-range, ordered patterns. The question is then to what extent the matrix structure drives the cell organization, and to what extent the cell behaviour patterns the matrix. It is difficult to observe in real time how the ECM changes when it interacts with cells, so the team turned to simulations, making use of a modified version of the classic Vicsek model of flocking. In the original Vicsek model, particles move around and interact by aligning with nearby particles. The modified model incorporated cells that could migrate and change each other's alignment, but added a new ingredient in the form of matrix fibres that both guided and were modified by the cells. Although cells that move independently of the fibres can generate certain matrix structures, the full range of patterns that are seen in vivo can only be generated if the matrix affects the cells. These results agreed with experiments that imaged cells and the ECM after the alignment processes took place.

The team then tested how these physical mechanisms were molecularly regulated and identified a transcription factor - a protein that regulates how genes are expressed that is needed for anisotropic matrices to form. Because an increase in matrix anisotropy can be associated with disease, drugs that prevent the matrix from becoming anisotropic may be beneficial. To identify candidates, the team cross-referenced their own data against publicly available datasets on how drugs affect gene expression. Six promising classes of compounds were identified, five of which had effects on ECM organization when tested.

Zoe Budrikis

ORIGINAL ARTICLE Park, D. et al. Extracellular matrix anisotropy is determined by TFAP2Cdependent regulation of cell collisions. Nat. Mater. https://doi.org/10.1038/s41563-019-0504-3 (2019).

RELATED ARTICLE Wershof, E. et al. Matrix feedback enables diverse higher-order patterning of the extracellular matrix. PLOS Comput. Biol. https://doi.org/10.1371/journal.pcbi.1007251 (2019). 